\title{
Perfect Omniscience, Perfect Secrecy and Steiner Tree Packing
}

\author{
Sirin Nitinawarat, Student Member, IEEE, and Prakash Narayan, Fellow, IEEE
}

\begin{abstract}
We consider perfect secret key generation for a "pairwise independent network" model in which every pair of terminals share a random binary string, with the strings shared by distinct terminal pairs being mutually independent. The terminals are then allowed to communicate interactively over a public noiseless channel of unlimited capacity. All the terminals as well as an eavesdropper observe this communication. The objective is to generate a perfect secret key shared by a given set of terminals at the largest rate possible, and concealed from the eavesdropper.

First, we show how the notion of perfect omniscience plays a central role in characterizing perfect secret key capacity. Second, a multigraph representation of the underlying secrecy model leads us to an efficient algorithm for perfect secret key generation based on maximal Steiner tree packing. This algorithm attains capacity when all the terminals seek to share a key, and, in general, attains at least half the capacity. Third, when a single "helper" terminal assists the remaining "user" terminals in generating a perfect secret key, we give necessary and sufficient conditions for the optimality of the algorithm; also, a "weak" helper is shown to be sufficient for optimality.

Index Terms - PIN model, perfect omniscience, perfect secret key, perfect secret key capacity, public communication, spanning tree packing, Steiner tree packing.
\end{abstract}

\section{INTRODUCTION}

Given a collection of terminals $\mathcal{M}=\{1, \ldots, m\}$, suppose that every pair $i, j$ of terminals, $1 \leq i<j \leq m$, share a random binary string of length $e_{i j}$ (bits), with the strings shared by distinct pairs of terminals being mutually independent. Then all the terminals are allowed to communicate interactively in multiple rounds over a public noiseless channel of unlimited capacity, with all such communication being observed by all the terminals. The main goal is to generate, for a given subset $A$ of the terminals in $\mathcal{M}$, a perfect secret key (SK) namely shared uniformly distributed random bits of the largest size - such that these shared bits are exactly independent of an eavesdropper's observations of the interterminal communication. All the terminals in $\mathcal{M}$ cooperate in generating such a perfect SK for $A$.

This model for perfect SK generation, hereafter referred to as a "pairwise independent network" (PIN) model, is a specialized version of an earlier PIN model [20], [19], [14]. In the latter, every pair of terminals observe a pair of correlated signals (not necessarily identical as here) that are independent

The work of S. Nitinawarat and P. Narayan was supported by the National Science Foundation under Grants CCF0515124, CCF0635271, CCF0830697 and InterDigital. The material in this paper was presented in part at the IEEE International Symposia on Information Theory, Toronto, Ontario, Canada, July 2008, and Seoul, Korea, June 2009.

The authors are with the Department of Electrical and Computer Engineering and the Institute for Systems Research, University of Maryland, College Park, MD 20742, USA.

Email: \{nitinawa, prakash\}@umd.edu of pairs of signals observed by all other terminal pairs. In [14], we had studied Shannon theoretic SK generation (not in the perfect sense) in the asymptotic limit of large signal observation lengths, and its connection to the combinatorial problem of Steiner tree packing of a multigraph. Leading work on Shannon theoretic SK generation with public communication originated in [9], [10], [1]; see also [2] for related models.

In contrast with [14], the present work bears the essence of "zero-error information theory," and accordingly, we rely on mathematical techniques of a combinatorial nature. Specifically, our emphasis here is on perfect SK generation for fixed signal observation lengths as well as for their asymptotic limits. For convenience, we shall continue to refer to our present model as the PIN model. This model possesses the appropriate structure for investigating the concept of perfect SK in which the generated key is exactly recoverable by every terminal in the secrecy seeking set $A$; is exactly independent of the eavesdropper's observations; and is uniformly distributed. Also, its special structure makes for a new concept of perfect omniscience, which plays a central role. Furthermore, in the spirit of [14], the PIN model reveals points of contact between perfect SK generation and the combinatorial problem of maximal Steiner tree packing of a multigraph. We remark that tree packing has been used in the context of network coding (see, for instance [7], [17]).

Our three main contributions described below are motivated by a known general connection between (not necessarily perfect) SK generation at the maximum rate and the minimum communication for (not necessarily perfect) omniscience [3], [4], and by the mentioned connection between the former and the combinatorial problem of maximal Steiner tree packing of a multigraph [14].

First, the concept of perfect omniscience enables us to obtain a single-letter formula for the perfect SK capacity of the PIN model; moreover, this capacity is shown to be achieved by linear noninteractive communication, and coincides with the (standard) SK capacity derived in our previous work [14]. This result establishes a connection between perfect SK capacity and the minimum rate of communication for perfect omniscience, thereby particularizing to the PIN model a known general link between these notions sans the requirement of the omniscience or secrecy being perfect [3].

Second, the PIN model can be represented by a multigraph. Taking advantage of this representation, we put forth an efficient algorithm for perfect SK generation using a maximal packing of Steiner trees of the multigraph. This algorithm involves public communication that is linear as well as noninteractive, and produces a perfect SK of length equal to the maximum size of such Steiner tree packing. When all the terminals in $\mathcal{M}$ seek to share a perfect SK, the algorithm is 
shown to achieve perfect SK capacity. However, when only a subset of terminals in $A \subset \mathcal{M}$ wish to share a perfect $\mathrm{SK}$, the algorithm can fall short of achieving capacity; nonetheless, it is shown to achieve at least half of it. Additionally, we obtain nonasymptotic and asymptotic bounds on the size and rate of the best perfect SKs generated by the algorithm. These bounds are of independent interest from a purely graph theoretic viewpoint as they constitute new estimates for the maximum size and rate of Steiner tree packing of a given multigraph.

Third, a special configuration of the PIN model arises when a lone "helper" terminal $m$ aids the "user" terminals in $A=\mathcal{M} \backslash\{m\}$ generate a perfect SK. This model has two special features: firstly, (a single) terminal $m$ possesses all the bit strings that are not in $A$; secondly, a Steiner tree for $A$ is a spanning tree for either $A$ or $\mathcal{M}$. These features enable us to obtain necessary and sufficient conditions for Steiner tree packing to achieve perfect SK capacity, as also a further sufficient condition that posits a "weak" role for the helper terminal $m$.

Preliminaries and the problem formulation are in Section II. Our results are described in Section III and proved in Section IV. A discussion follows in Section V.

\section{PRELIMINARIES}

Suppose that the terminals in $\mathcal{M}=\{1, \ldots, m\}, m \geq$ 2 , observe, respectively, $n$ independent and identically distributed (i.i.d.) repetitions of the $\operatorname{rvs} \tilde{X}_{1}, \ldots, \tilde{X}_{m}$, denoted by $\tilde{X}_{1}^{n}, \ldots, \tilde{X}_{m}^{n}$, where $\tilde{X}_{i}^{n}=\left(\tilde{X}_{i, 1}, \ldots, \tilde{X}_{i, n}\right), i \in \mathcal{M}$. We shall be concerned throughout with a PIN model $\tilde{X}_{1}, \ldots, \tilde{X}_{m}$ [19], defined by each $\operatorname{rv} \tilde{X}_{i}, i \in \mathcal{M}$, being of the form $\tilde{X}_{i}=\left(X_{i j}, j \in \mathcal{M} \backslash\{i\}\right)$ with $m-1$ components, and the "reciprocal pairs" of rvs $\left\{\left(X_{i j}, X_{j i}\right), 1 \leq i<j \leq m\right\}$ being mutually independent. We assume further that $X_{i j}=$ $X_{j i}, 1 \leq i \neq j \leq m$, where $X_{i j}$ is uniformly distributed over the set of all binary strings of length $e_{i j}$ (bits). Thus, every pair of terminals is associated with a random binary string that is independent of all other random binary strings associated with all other pairs of terminals. The assumption is tantamount to every pair of terminals $i, j$ sharing at the outset privileged and pairwise "perfect secrecy" of $e_{i j}$ bits. Following their observation of the random sequences as above, the terminals in $\mathcal{M}$ are allowed to communicate among themselves over a public noiseless channel of unlimited capacity; all such public communication, which maybe interactive and conducted in multiple rounds, is observed by all the terminals. A communication from a terminal, in general, can be any function of its observed sequence as well as all previous public communication. The public communication of all the terminals will be denoted collectively by $\mathbf{F}=\mathbf{F}^{(n)}$.

Definition 1: The communication $\mathbf{F}$ is termed linear noninteractive communication (LC) if $\mathbf{F}=\left(F_{1}, \ldots, F_{m}\right)$ with $F_{i}=L_{i} \tilde{X}_{i}^{n}$, where $L_{i}$ is a $b_{i} \times\left(\sum_{j \neq i} n e_{i j}\right)$ matrix with $\{0,1\}$-valued entries, $i=1, \ldots, m$. The integer $b_{i} \geq 0, i=1, \ldots, m$, represents the length (in bits) of the

\footnotetext{
${ }^{1}$ All additions and multiplications are modulo 2 .
}

${ }^{2}$ It is assumed that $\sum_{j \neq i} e_{i j} \geq 1, \quad i=1, \ldots, m$. communication $F_{i}$ from terminal $i$; the overall communication $\mathbf{F}$ has length $\sum_{i=1}^{m} b_{i}$ (bits).

The primary goal is to generate shared perfect secret common randomness for a given set $A \subseteq \mathcal{M}$ of terminals at the largest rate possible, with the remaining terminals (if any) cooperating in secrecy generation. The resulting perfect secret key must be accessible to every terminal in $A$; but it need not be accessible to the terminals not in $A$ and nor does it need to be concealed from them. It must, of course, be kept perfectly secret from the eavesdropper that has access to the public interterminal communication $\mathbf{F}$, but is otherwise passive, i.e., unable to tamper with this communication.

The following basic concepts and definitions are adapted from [3], [4]. For rvs $U, V$, we say that $U$ is perfectly recoverable from $V$ if $\operatorname{Pr}\{U=f(V)\}=1$ for some function $f(V)$. With the rvs $K$ and $\mathbf{F}$ representing a secret key and the eavesdropper's knowledge, respectively, information theoretic perfect secrecy entails that the security index

$$
\begin{aligned}
s(K ; \mathbf{F}) & =\log |\mathcal{K}|-H(K)+I(K \wedge \mathbf{F}) \\
& =\log |\mathcal{K}|-H(K \mid \mathbf{F})=0,
\end{aligned}
$$

where $\mathcal{K}$ is the range of $K$ and $|\cdot|$ denotes cardinality. This requirement simultaneously renders $K$ to be uniformly distributed and independent of $\mathbf{F}$.

Definition 2: Given any set $A \subseteq \mathcal{M}$ of size $|A| \geq 2$, a rv $K$ is a perfect secret key (SK) for the set of terminals $A$ achievable with communication $\mathbf{F}$, if $K$ is perfectly recoverable 4 from $\left(\tilde{X}_{i}^{n}, \mathbf{F}\right)$ for each $i \in A$ and, in addition, it satisfies the perfect secrecy condition (1).

Definition 3: A number $R$ is an achievable perfect $S K$ rate for a set of terminals $A \subseteq \mathcal{M}$ if there exist perfect SKs $K^{(n)}$ for $A$ achievable with appropriate communication, such that

$$
\frac{1}{n} \log \left|\mathcal{K}^{(n)}\right| \rightarrow R \quad \text { as } \quad n \rightarrow \infty,
$$

where $\mathcal{K}^{(n)}$ is the range of $K^{(n)}$. The largest achievable perfect SK rate is the perfect SK capacity $C(A)$.

Thus, by definition, the perfect SK capacity for $A$ is the largest rate of a rv that is perfectly recoverable at each terminal in $A$ from the aggregate information available to it, and is uniformly distributed and concealed from an eavesdropper with access to the public interterminal communication; it need not be concealed from the terminals in $A^{c}=\mathcal{M} \backslash A$, which cooperate in secrecy generation. The notion of perfect SK capacity is more stringent than that of SK capacity under the requirements of the key being asymptotically recoverable for each $i \in A$ and the security index tending to 0 , both as $n \rightarrow \infty$; in particular, now the security index must equal zero for all sufficiently large $n$. The latter SK capacity for the PIN model has been characterized in [12], [13], [14].

A central role is played by the notion of perfect omniscience which is a strict version of the concept of omniscience introduced in [3]. This notion does not involve any secrecy requirements.

\footnotetext{
${ }^{3}$ All logarithms are to the base 2 .

4 The extra requirement of perfectness in recoverability is not a limiting factor for the PIN model in contrast with other models of SK generation.
} 
Definition 4: The communication $\mathbf{F}$ is communication for perfect omniscience for $A$ if $\left(\tilde{X}_{1}^{n}, \ldots, \tilde{X}_{m}^{n}\right)$ is perfectly recoverable from $\left(\tilde{X}_{i}^{n}, \mathbf{F}\right)$ for every $i \in A$. Further, $\mathbf{F}$ is linear noninteractive communication for perfect omniscience $\left(\mathrm{LCO}^{(n)}(A)\right)$ if $\mathbf{F}$ is an LC and satisfies the previous perfect recoverability condition. The minimum length (in bits) of an $\mathrm{LCO}^{(n)}(A)$, i.e., $\min _{\mathrm{LCO}^{(n)}(A)} \sum_{i=1}^{m} b_{i}$, will be denoted by $\mathrm{LCO}_{m}^{(n)}(A)$. The minimum rate of $\mathrm{LCO}^{(n)}(A)$ is $O M N(A) \triangleq$ $\lim \sup _{n} \frac{1}{n} \mathrm{LCO}_{m}^{(n)}(A)$.

\section{RESULTS}

\section{A. Perfect SK Capacity for the PIN Model}

Our first main contribution is a (single-letter) characterization of the perfect SK capacity for the PIN model, which brings forth a connection with the minimum rate of communication for perfect omniscience.

Theorem 1: The perfect $S K$ capacity for a set of terminals $A \subseteq \mathcal{M}$ is

$$
C(A)=\sum_{i, j} e_{i j}-O M N(A)
$$

where

$$
O M N(A)=\min _{\left(R_{1}, \ldots, R_{m}\right) \in \mathcal{R}(A)} \sum_{i=1}^{m} R_{i},
$$

with

$$
\begin{aligned}
& \mathcal{R}(A)= \\
& \left\{\begin{array}{c}
\left(R_{1}, \ldots, R_{m}\right) \in \mathbb{R}^{m}: R_{i} \geq 0, i=1, \ldots, m, \\
\sum_{i \in B} R_{i} \geq \sum_{1 \leq i<j \leq m, i \in B, j \in B} e_{i j}, \\
\forall B \nsupseteq A, \emptyset \neq B \subset \mathcal{M}
\end{array}\right\} .
\end{aligned}
$$

Furthermore, this perfect SK capacity can be achieved with linear noninteractive communication.

Remarks: (i) Clearly, the perfect SK capacity, by definition, cannot exceed the (standard) SK capacity studied in [12], [14]. Indeed, Theorem 1 implies that the latter is attained by a perfect SK.

(ii) In the same vein, the minimum rate of communication for (asymptotic) omniscience [3] can be attained for the PIN model with perfect recoverability at $A$ of $\left(\tilde{X}_{1}^{n}, \ldots, \tilde{X}_{m}^{n}\right)$ for all $n$ sufficiently large, and with linear noninteractive communication. We mention that noninteractive communication, without a claim of linearity, was shown to suffice for (asymptotic) omniscience in [3].

\section{B. Maximal Steiner Tree Packing and Perfect SK Generation}

Theorem 1 serves to establish the sufficiency of an LC in achieving perfect SK capacity through the intermediate attainment of perfect omniscience for $A$, as seen in its proof below. However, as also evident from the proof, decoding is by exhaustive search of prohibitive complexity.

The PIN model can be represented by a multigraph. This representation leads us to an efficient algorithm for perfect SK generation, not necessarily through perfect omniscience, by a maximal packing of Steiner trees of the multigraph.
In particular, this algorithm will be seen to entail public communication in the form of an LC. On the other hand, such an algorithm based on maximal Steiner tree packing need not attain perfect SK capacity. The size of the largest perfect SK that is thus generated can be estimated in terms of the minimum length of an $\operatorname{LCO}^{(n)}(A)$.

Definition 5: A multigraph $G=(V, E)$ with vertex set $V$ and edge set $E$ is a connected undirected graph with no selfloops and with multiple edges possible between any pair of vertices. Given $G=(V, E)$ and a positive integer $n$, let $G^{(n)}=\left(V, E^{(n)}\right)$ denote the multigraph with vertex set $V$ and edge set $E^{(n)}$ wherein every vertex pair is connected by $n$ times as many edges as in $E$; in particular, $G^{(1)}=G$. Furthermore, $\left|E^{(n)}\right|$ will denote the total number of edges in $E^{(n)}$.

To the PIN model $\tilde{X}_{1}, \ldots, \tilde{X}_{m}$ (cf. section II), we can associate a multigraph $G=(\mathcal{M}, E)$ with $\mathcal{M}=\{1, \ldots, m\}$ and the number of edges connecting a vertex pair $(i, j)$ in $E$ equal to $e_{i j}$; in particular, the edge connecting $(i, j)$ will be associated with the random binary string $X_{i j}$.

By this association, it will be convenient to represent (3) and (4) as

$$
O M N_{G}(A)=\min _{\left(R_{1}, \ldots, R_{m}\right) \in \mathcal{R}_{G}(A)} \sum_{i=1}^{m} R_{i},
$$

with

$$
\begin{aligned}
& \mathcal{R}_{G}(A)= \\
& \left\{\begin{array}{c}
\left(R_{1}, \ldots, R_{m}\right) \in \mathbb{R}^{m}: R_{i} \geq 0, i=1, \ldots, m, \\
\sum_{i \in B} R_{i} \geq \sum_{1 \leq i<j \leq m, i \in B, j \in B} e_{i j}, \\
\forall B \nsupseteq A, \emptyset \neq B \subset \mathcal{M}
\end{array}\right\},
\end{aligned}
$$

whereupon (2) can be restated as

$$
C(A)=|E|-O M N_{G}(A) .
$$

Furthermore, it is easy and useful to note that for every $n \geq 1$,

$$
O M N_{G^{(n)}}(A)=n O M N_{G}(A) .
$$

Definition 6: For $A \subseteq V$, a Steiner tree (for $A$ ) of $G=$ $(V, E)$ is a subgraph of $G$ that is a tree, i.e., containing no cycle, and whose vertex set contains $A$; such a Steiner tree is said to cover A. A Steiner tree packing of $G$ is any collection of edge-disjoint Steiner trees of $G$. Let $\mu(A, G)$ denote the maximum size of such a packing (cf. [5]), i.e., the maximum number of trees in the packing. The maximum rat 5 of Steiner tree packing of $G$ is $\limsup _{n \rightarrow \infty} \frac{1}{n} \mu\left(A, G^{(n)}\right)$. When $A=V$, a Steiner tree becomes a spanning tree, with corresponding notions of spanning tree packing, maximum size and rate.

Given a PIN model, the notion of Steiner tree packing of the associated multigraph leads to an efficient algorithm for constructing an $\mathrm{LCO}^{(n)}(A)$ and thereby generating a perfect SK. The next Theorem 2 indicates that the largest size of a perfect SK that the algorithm generates is the maximum size of the Steiner tree packing. Furthermore, Theorem 2 and its corollary, and Theorem 5 provide nonasymptotic and asymptotic bounds on the size and rate, respectively, of the

\footnotetext{
${ }^{5}$ In fact, $\lim _{n \rightarrow \infty} \frac{1}{n} \mu\left(A, G^{(n)}\right)$ exists, as shown later in Proposition 4.
} 
best perfect SKs generated by the algorithm. Of independent interest from a purely graph theoretic viewpoint, these results also constitute new bounds for the maximum size and rate of Steiner tree packing of a given multigraph.

Theorem 2: For the multigraph $G=(\mathcal{M}, E)$ associated with a PIN model and for $A \subseteq \mathcal{M}$, it holds for every $n \geq 1$ that

(i) the terminals in $\mathcal{M}$ can devise an $\operatorname{LCO}^{(n)}(A)$ of total length $n\left|E^{(1)}\right|-\mu\left(A, G^{(n)}\right)$ and subsequently generate a perfect $S K \quad K^{(n)}$ with $\log \left|\mathcal{K}^{(n)}\right|=\mu\left(A, G^{(n)}\right)$;

(ii) $\mu\left(A, G^{(n)}\right) \leq n\left|E^{(1)}\right|-L C O_{m}^{(n)}(A)$;

(iii) furthermore, $\operatorname{LCO}_{m}^{(n)}(A)$ is bounded below by the value of an integer linear program according to

$$
\operatorname{LCO}_{m}^{(n)}(A) \geq I N T_{G^{(n)}}(A)
$$

where

$$
I N T_{G^{(n)}}(A)=\min _{\left(I_{1}, \ldots, I_{m}\right) \in \mathcal{I}_{G^{(n)}}(A)} \sum_{i=1}^{m} I_{i},
$$

with

$$
\begin{aligned}
& \mathcal{I}_{G^{(n)}}(A)= \\
& \left\{\begin{array}{c}
\left(I_{1}, \ldots, I_{m}\right) \in \mathbb{Z}^{m}: I_{i} \geq 0, i=1, \ldots, m, \\
\sum_{i \in B} I_{i} \geq n \sum_{1 \leq i<j \leq m, i \in B, j \in B} e_{i j}, \\
\forall B \nsupseteq A, \bar{\emptyset} \neq \boldsymbol{D} \subset \mathcal{M}
\end{array}\right\} .
\end{aligned}
$$

Corollary 3: For every $n \geq 1$, the maximum size of Steiner tree packing of a multigraph $G^{(n)}$ satisfies

$$
\mu\left(A, G^{(n)}\right) \leq n\left|E^{(1)}\right|-I N T_{G^{(n)}}(A),
$$

with equality when $A=\mathcal{M}$.

Remarks: (i) Note that the bounds in Theorem 2 are nonasymptotic, i.e., valid for every $n$. Also, note in the bound in Theorem 2 (ii) for $\mu\left(A, G^{(n)}\right)$ that $\operatorname{LCO}_{m}^{(n)}(A)$ is defined in terms of its operational significance.

(ii) Further, Theorem 2 provides a nonasymptotic computable lower bound for $\operatorname{LCO}_{m}^{(n)}(A)$ in terms of an integer linear program. The optimum value of its linear programming relaxation constitutes a further lower bound which equals $O M N_{G^{(n)}}(A)=n O M N_{G}(A)$, by (8).

Next, we turn to connections between perfect SK capacity $C(A)$ and the maximum rate of Steiner tree packing of $G=$ $(\mathcal{M}, E)$. The following concept of "fractional" Steiner tree packing will be relevant.

For $A \subseteq \mathcal{M}=\{1, \ldots, m\}$, consider the collection $\left\{S_{1}, \ldots, S_{k}\right\}$ of all distinct Steiner trees (for $A$ ) of $G$, where $k=k(G)$. Consider the region

$$
\begin{aligned}
& \mathcal{T}_{G}(A)= \\
& \left\{\begin{array}{c}
\left(T_{1}, \ldots, T_{k}\right) \in \mathbb{R}^{k}: T_{l} \geq 0, l=1, \ldots, k, \\
\sum_{l:(i, j) \in S_{l}} T_{l} \leq e_{i j} \\
\forall(i, j), 1 \leq i<j \leq m
\end{array}\right\} .
\end{aligned}
$$

Definition 7: For a multigraph $G=(\mathcal{M}, E)$ and $A \subseteq \mathcal{M}$, the maximal "fractional" Steiner tree packing of $G$, denoted
$\mu_{f}(A, G)$, is $\mu_{f}(A, G) \triangleq \max _{\mathcal{T}_{G}(A)} \sum_{l=1}^{k} T_{l}$.

Remarks: (i) Clearly, $\mu_{f}(A, G)$ corresponds to a linear program with finite optimum value, and the maximum is attained. Furthermore, it is readily verified that for every $n \geq 1$

$$
\mu_{f}\left(A, G^{(n)}\right)=n \mu_{f}(A, G) .
$$

(ii) We observe that in Definition 6, $\mu(A, G) \triangleq$ $\max _{\mathcal{T}_{G}(A) \cap \mathbb{Z}^{k}} \sum_{l=1}^{k} T_{l}$.

Proposition 4: For a multigraph $G=(\mathcal{M}, E)$ and $A \subseteq \mathcal{M}$, it holds that the maximum rate of Steiner tree packing (for $A$ ) of $G$ satisfies

$$
\begin{aligned}
\limsup _{n \rightarrow \infty} \frac{1}{n} \mu\left(A, G^{(n)}\right) & =\liminf _{n \rightarrow \infty} \frac{1}{n} \mu\left(A, G^{(n)}\right) \\
& =\lim _{n \rightarrow \infty} \frac{1}{n} \mu\left(A, G^{(n)}\right) \\
& =\mu_{f}(A, G) .
\end{aligned}
$$

Theorem 5: For the multigraph $G=(\mathcal{M}, E)$ associated with the PIN model and for $A \subseteq \mathcal{M}$, it holds that

$$
\frac{1}{2} C(A) \leq \lim _{n \rightarrow \infty} \frac{1}{n} \mu\left(A, G^{(n)}\right) \leq C(A) .
$$

Furthermore, when $A=\mathcal{M}$,

$$
\lim _{n \rightarrow \infty} \frac{1}{n} \mu\left(\mathcal{M}, G^{(n)}\right)=C(\mathcal{M}) .
$$

Remark: For the PIN model with $m$ terminals, every Steiner tree has at most $m-1$ edges. Also, from (16), $\mu\left(A, G^{(n)}\right) \lesssim n C(A)$ for all large $n$. Hence, the overall complexity of the perfect SK generation algorithm based on Steiner tree packing is linear (in $n$ ).

The upper bound on $\lim _{n \rightarrow \infty} \frac{1}{n} \mu\left(A, G^{(n)}\right)$ in Theorem 5 is not tight, in general, as seen by the following example.

Example: Consider the multigraph [7] in Figure 1 with $|\mathcal{M}|=7$ and $|A|=4$; the terminals in $A$ are represented by the solid circles and every shown edge is single. Computations give that $C(A)=2.0$ by (7), (5), while $\lim _{n \rightarrow \infty} \frac{1}{n} \mu\left(G^{(n)}, A\right)=1.8$ by Proposition 4 and the scheme in Lemma 1.

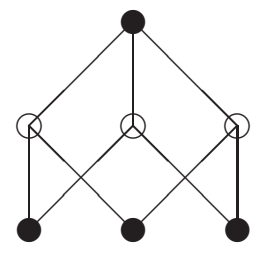

Figure 1: Example

\section{The Single Helper Case}

As observed after Theorem 5, the maximum rate of Steiner tree packing can fail to achieve perfect SK capacity. A natural question that remains open is whether the maximum rate of Steiner tree packing equals perfect SK capacity for the special case of the PIN model in which a lone "helper" terminal $m$ 
assists the "user" terminals in $A=\{1, \ldots, m-1\}$ generate a perfect SK. In this section, we provide partial answers.

First, we derive necessary and sufficient conditions for the maximum rate of Steiner tree packing to equal perfect SK capacity in (16) and, analogously, the (nonasymptotic) maximum size of Steiner tree packing to meet its upper bound in (12). These conditions entail the notion of a fractional multigraph. Throughout this section, we shall assume that $A=\{1, \ldots, m-1\} \subset \mathcal{M}=\{1, \ldots, m\}$.

Definition 8: Given a multigraph $G=(\mathcal{M}, E)$ as in Definition 5, a fractional multigraph $\tilde{G}=(A, \tilde{E})$ in $A$ (with vertex set $A$ ) has edge set $\tilde{E}=\left\{\tilde{e}_{i j} \in \mathbb{R}, 0 \leq \tilde{e}_{i j} \leq e_{i j}, 1 \leq\right.$ $i<j \leq m-1\}$. For any such $\tilde{G}$, the complementary fractional multigraph $G \backslash \tilde{G}=(\mathcal{M}, E \backslash \tilde{E})$ has vertex set $\mathcal{M}$ and edge set $E \backslash \tilde{E} \triangleq\left\{e_{i j}-\tilde{e}_{i j}, 1 \leq i<j \leq m-1 ; e_{i m}, 1 \leq i \leq m-1\right\}$. The definitions of $\mathcal{R}_{G}(A)$ in (6), $O M N_{G}(A)$ in [5], $\mathcal{T}_{G}(A)$ in (13) and $\mu_{f}(A, G)$ in Definition 7 all have obvious extensions to $\tilde{G}$ and $G \backslash \tilde{G}$ as well. Further, (8) and (14) also hold for $\tilde{G}$ and $G \backslash \tilde{G}$.

Proposition 6: For the multigraph $G=(\mathcal{M}, E)$ associated with the PIN model, the following hold:

(i)

(ii)

$$
\mu_{f}(A, G) \geq \max _{\tilde{G}} \mu_{f}(A, \tilde{G})+\mu_{f}(\mathcal{M}, G \backslash \tilde{G})
$$

$$
O M N_{G}(A) \leq \min _{\tilde{G}} O M N_{\tilde{G}}(A)+O M N_{G \backslash \tilde{G}}(\mathcal{M}) ;
$$

(iii)

$$
\mu(A, G) \geq \max _{\tilde{G}_{I}} \mu\left(A, \tilde{G}_{I}\right)+\mu\left(\mathcal{M}, G \backslash \tilde{G}_{I}\right)
$$

(iv)

$$
I N T_{G}(A) \leq \min _{\tilde{G}_{I}} I N T_{\tilde{G}_{I}}(A)+I N T_{G \backslash \tilde{G}_{I}}(\mathcal{M}),
$$

where the optima in (i) and (ii) are over all fractional multigraphs $\tilde{G}=(A, \tilde{E})$ in $A$, and the optima in (iii) and (iv) are over all multigraphs $\tilde{G}_{I}=(A, \tilde{E})$ in $A$ for which $\tilde{E}$ consists of only integer-valued $\tilde{e}_{i j}$ s.

Theorem 7: For the multigraph $G=(\mathcal{M}, E)$ associated with the PIN model,

(i)

$$
\lim _{n \rightarrow \infty} \frac{1}{n} \mu\left(A, G^{(n)}\right)=C(A)
$$

iff

$$
O M N_{G}(A)=\min _{\tilde{G}} O M N_{\tilde{G}}(A)+O M N_{G \backslash \tilde{G}}(\mathcal{M}),
$$

where the minimum is over all fractional multigraphs $\tilde{G}=$ $(A, \tilde{E})$ in $A$;

(ii)

$$
\mu\left(A, G^{(n)}\right)=|E|-I N T_{G}(A)
$$

iff

$$
I N T_{G}(A)=\min _{\tilde{G}_{I}} I N T_{\tilde{G}_{I}}(A)+I N T_{G \backslash \tilde{G}_{I}}(\mathcal{M}),
$$

where the minimum is over all multigraphs $\tilde{G}_{I}=(A, \tilde{E})$ for which $\tilde{E}$ consists of only integer-valued $\tilde{e}_{i j} s$.

Our final result provides another sufficient condition for the maximum rate of Steiner tree packing to equal perfect SK capacity. Recall from Theorem 1 that, in general, perfect SK capacity for $A$ can be attained with public communication that corresponds to the minimum communication for perfect omniscience. If the latter can be accomplished with the sole helper terminal $m$ communicating "sparingly," then it transpires that maximal Steiner tree packing attains the best perfect SK rate. An analogous nonasymptotic version of this claim also holds. Heuristically, a sufficient "weak" role of the helper terminal $m$ turns the Steiner tree packing of $A$, in effect, into a spanning tree packing of $A$.

Let $d_{i} \triangleq \sum_{j \neq i} e_{i j}$ denote the degree of vertex $i, i \in \mathcal{M}$. Clearly, any $\left(R_{1}^{*}, \ldots, R_{m}^{*}\right)$ (resp. $\left.\left(I_{1}^{*}, \ldots, I_{m}^{*}\right)\right)$ that attains the minimum corresponding to $O M N_{G}(A)$ (cf. (5)) (resp. $I N T_{G}(A)$ (cf. (10)) must satisfy $R_{i}^{*} \leq d_{i}\left(\right.$ resp. $\left.I_{i}^{*} \leq d_{i}\right)$, $i=1, \ldots, m$.

Theorem 8: For the multigraph $G=(\mathcal{M}, E)$ associated with the PIN model,

(i) if there exists $\left(R_{1}^{*}, \ldots, R_{m}^{*}\right)$ that attains $O M N_{G}(A)$ (cf. (5)) with $R_{m}^{*} \leq d_{m} / 2$, then

$$
\lim _{n \rightarrow \infty} \frac{1}{n} \mu\left(A, G^{(n)}\right)=C(A)=|E|-O M N_{G}(A) .
$$

(ii) if there exists $\left(I_{1}^{*}, \ldots, I_{m}^{*}\right)$ that attains $I N T_{G}(A)$ (cf. (10)) with $I_{m}^{*} \leq\left\lfloor d_{m} / 2\right\rfloor$, then

$$
\mu(A, G)=|E|-I N T_{G}(A) .
$$

\section{PROOFS}

Proof of Theorem 1: From remark (i) following Theorem 1 , we need prove only the achievability part. The main step is to show, using a random coding argument, the existence with large probability of an $\mathrm{LCO}^{(n)}(A)$ of small length under appropriate conditions; the terminals in $A$ then extract from the corresponding perfect omniscience a perfect SK of optimum rate.

Let $\tilde{X}_{\mathcal{M}}^{n}=\left(\tilde{X}_{1}^{n}, \ldots, \tilde{X}_{m}^{n}\right)$ take values in $\tilde{\mathcal{X}}_{\mathcal{M}}^{n}=\tilde{\mathcal{X}}_{1}^{n} \times$ $\ldots \times \tilde{\mathcal{X}}_{m}^{n}$, where $\tilde{\mathcal{X}}_{i}^{n}=\{0,1\}^{\sum_{j \neq i} n e_{i j}}$. We denote a realization of $\tilde{X}_{\mathcal{M}}^{n}$ by $\tilde{x}_{\mathcal{M}}^{n}=\left(\tilde{x}_{1}^{n}, \ldots, \tilde{x}_{m}^{n}\right)$. Fix $b_{1}, \ldots, b_{m}$. Let $\mathbf{L}=\left(\mathbf{L}_{1}, \ldots, \mathbf{L}_{m}\right)$ consist of mutually independent random matrices of appropriate dimensions as in Definition 1. Furthermore, the $\mathbf{r v} \mathbf{L}_{i}$ consists of i.i.d. equiprobable components, $i=1, \ldots, m$. Clearly, $\mathbf{L}_{1}, \ldots, \mathbf{L}_{m}$ makes for a random $\mathbf{L C}$.

Since for $\mathbf{L}_{1}, \ldots, \mathbf{L}_{m}$ to constitute an $\operatorname{LCO}^{(n)}(A)$, it suffices that the mapping

$$
\tilde{x}_{\mathcal{M}}^{n} \rightarrow\left(\tilde{x}_{i}^{n}, \mathbf{L}_{1} \tilde{x}_{1}^{n}, \ldots, \mathbf{L}_{m} \tilde{x}_{m}^{n}\right)
$$

be one-to-one for every $i \in A$, we have

$\operatorname{Pr}\left\{\mathbf{L}\right.$ does not constitute an $\left.\mathrm{LCO}^{(n)}(A)\right\}$ 


$$
\begin{aligned}
& =\operatorname{Pr}\left\{\begin{array}{c}
\exists \tilde{x}_{\mathcal{M}}^{n} \neq \tilde{y}_{\mathcal{M}}^{n} \in \tilde{\mathcal{X}}_{\mathcal{M}}^{n} \text { satisfying } \\
\tilde{x}_{j}^{n}=\tilde{y}_{j}^{n} \text { for some } j \in A \text { such that } \\
\mathbf{L}_{i} \tilde{x}_{i}^{n}=\mathbf{L}_{i} \tilde{y}_{i}^{n} \text { for each } i=1, \ldots, m
\end{array}\right\} \\
& =\operatorname{Pr}\left\{\begin{array}{c}
\exists \tilde{x}_{\mathcal{M}}^{n} \neq \mathbf{0} \in \tilde{\mathcal{X}}_{\mathcal{M}}^{n} \text { satisfying } \\
\tilde{x}_{j}^{n}=\mathbf{0} \text { for some } j \in A \text { such that } \\
\mathbf{L}_{i} \tilde{x}_{i}^{n}=\mathbf{0} \text { for each } i=1, \ldots, m
\end{array}\right\} \\
& \leq \sum_{B \neq \emptyset,}^{\exists} \operatorname{Pr}\left\{\begin{array}{c}
\exists \tilde{x}_{\mathcal{M}}^{n} \in \tilde{\mathcal{X}}_{\mathcal{M}}^{n} \text { satisfying } \\
\tilde{x}_{j}^{n} \neq \mathbf{0} \forall j \in B, \text { and } \tilde{x}_{j}^{n}=\mathbf{0} \forall j \in B^{c} \\
\text { such that } \mathbf{L}_{i} \tilde{x}_{i}^{n}=\mathbf{0} \\
\text { for each } i=1, \ldots, m
\end{array}\right\},
\end{aligned}
$$

where 210 is by the linearity of the communication and 22 is obtained by applying the union bound to the event in 21.

Now, we note by the assumed independence of $\mathbf{L}_{1}, \ldots \mathbf{L}_{m}$ and the fact that the components of $\mathbf{L}_{i}$ are i.i.d. and equiprobable, $i=1, \ldots, m$, that for each nonempty $B \nsupseteq A$, and any $\tilde{x}_{\mathcal{M}}^{n}$ satisfying $\tilde{x}_{j}^{n} \neq \mathbf{0} \forall j \in B$, and $\tilde{x}_{j}^{n}=\mathbf{0} \forall j \in B^{c}$, we have

$$
\begin{aligned}
\operatorname{Pr}\left\{\mathbf{L}_{i} \tilde{x}_{i}^{n}=\right. & \mathbf{0} \text { for every } i=1, \ldots, m\} \\
& =\operatorname{Pr}\left\{\mathbf{L}_{i} \tilde{x}_{i}^{n}=\mathbf{0} \text { for every } i \in B\right\} \\
& =\prod_{i \in B} 2^{-b_{i}}=2^{-\sum_{i \in B} b_{i}}
\end{aligned}
$$

Continuing with (22) upon using (23), we obtain

$\operatorname{Pr}\left\{\mathbf{L}\right.$ does not constitute an $\left.\operatorname{LCO}^{(n)}(A)\right\}$

$$
\begin{aligned}
& \leq \sum_{\substack{B \neq \emptyset, B \nsupseteq A}}\left|\left\{\begin{array}{l}
\tilde{x}_{\mathcal{M}}^{n} \in \tilde{\mathcal{X}}_{\mathcal{M}}^{n}: \tilde{x}_{j}^{n} \neq \mathbf{0} \\
\forall j \in B, \tilde{x}_{j}^{n}=\mathbf{0} \forall j \in B^{c}
\end{array}\right\}\right| 2^{-\sum_{i \in B} b_{i}} \\
& \leq \sum_{\substack{B \neq \emptyset \\
B \nsupseteq A}} 2^{n\left(\sum_{l, k \in B} e_{l k}\right)} 2^{-\sum_{i \in B} b_{i}} \\
& =\sum_{\substack{B \neq \emptyset \\
B \nsupseteq A}} 2^{-n\left(\frac{1}{n} \sum_{i \in B} b_{i}-\sum_{l, k \in B} e_{l k}\right)}
\end{aligned}
$$

We note that in this proof, the special structure of the PIN model is used for the first time in the second inequality above.

Now, let $\left(R_{1}^{*}, \ldots, R_{m}^{*}\right)$ achieve the minimum in the rightside of (3). Pick an arbitrary $\epsilon>0$ and choose $b_{i}$ in (24) as $b_{i}=\left\lceil n\left(R_{i}^{*}+\epsilon\right)\right\rceil, i=1, \ldots, m$. Then, by the definition of $\mathcal{R}(A)$, the right side of 24 decays to zero exponentially rapidly in $n$; in particular, we get from that for all $n$ sufficiently large, $\mathbf{L}$ constitutes an $\mathrm{LCO}^{(n)}(A)$ with large probability. This implies the existence of a (deterministic) $L=\left(L_{1}, \ldots, L_{m}\right)$ that constitutes an $\operatorname{LCO}^{(n)}(A)$ for all $n$ sufficiently large.

It remains to extract a perfect SK from the perfect omniscience obtained above. By the definition of the PIN model, observe that

$$
\operatorname{Pr}\left\{\tilde{X}_{\mathcal{M}}^{n}=\tilde{x}_{\mathcal{M}}^{n}\right\}=2^{-\sum_{l, k} n e_{l k}} \text { for all } \tilde{x}_{\mathcal{M}}^{n} \in \tilde{\mathcal{X}}_{\mathcal{M}}^{n} .
$$

By the linearity of the $\operatorname{LCO}^{(n)}(A)$ above, it is readily seen that

the cardinality $\left|\left\{\tilde{x}_{\mathcal{M}}^{n} \in \tilde{\mathcal{X}}_{\mathcal{M}}^{n}: L_{i} \tilde{x}_{i}^{n}=a_{i}, i=1, \ldots, m\right\}\right|$ is the same for all feasible $\left(a_{1}, \ldots, a_{m}\right)$ where $a_{i} \in\{0,1\}^{b_{i}}, i=$ $1, \ldots, m$, and that this common number is at least

$$
N=2^{\left(\sum_{l, k} n e_{l k}\right)-\left(\sum_{i=1}^{m} b_{i}\right)} \text {. }
$$

For each communication message $\left(a_{1}, \ldots, a_{m}\right)$, we index the elements of the coset $\left\{\tilde{x}_{\mathcal{M}}^{n}: L_{i} \tilde{x}_{i}^{n}=a_{i}, i=1, \ldots, m\right\}$ in a fixed manner. Then, for a realization $\tilde{x}_{\mathcal{M}}^{n} \in \tilde{\mathcal{X}}_{\mathcal{M}}^{n}$, every terminal in $A$ (which knows $\tilde{x}_{\mathcal{M}}^{n}$ by omniscience) picks as the perfect SK the index of $\tilde{x}_{\mathcal{M}}^{n}$ in its coset, as in [18]. Since $\tilde{X}_{\mathcal{M}}^{n}$ takes values in $\tilde{\mathcal{X}}_{\mathcal{M}}^{n}$ and since each coset has the same size, it follows that this random index is uniformly distributed and independent of the coset (the communication message), thereby constituting a perfect SK. Lastly, the rate of this perfect SK is at least

$$
\begin{aligned}
\lim _{n \rightarrow \infty} \frac{1}{n} \log N & =\sum_{l, k} e_{l k}-\sum_{i=1}^{m} R_{i}^{*}-m \epsilon \\
& =\sum_{l, k} e_{l k}-\operatorname{OMN}(A)-m \epsilon,
\end{aligned}
$$

where $\epsilon>0$ is arbitrary.

Proof of Theorem 2: The proof will rely on the technical Lemma 1 which is stated next and established in Appendix A.

Lemma 1: Let $G=(V, T)$ be a tree, and associate with each edge a bit. Then the terminals in $V$ can devise a (noninteractive) LC of length $|T|-1$ bits enabling every terminal in $V$ to recover all the edges of $T$, i.e., all the bits associated with the edges of $T$.

(i,ii) If $\mu\left(A, G^{(n)}\right)=k$, say, then $E^{(n)}$ is the disjoint union of $k$ Steiner trees $T_{1}, \ldots, T_{k}$ (each of which covers $A$ ) and the remaining edge set $R$, so that

$$
\left|E^{(n)}\right|=n\left|E^{(1)}\right|=\sum_{i=1}^{k}\left|T_{i}\right|+|R|,
$$

where $\left|T_{i}\right|$ denote the number of edges in $T_{i}$.

Apply Lemma 1 to every Steiner tree $T_{i}, i=1, \ldots, k$, in (25) to get $k$ LCs that enable every terminal in $A$ to recover the edges of all the $T_{i}, i=1, \ldots, k$. An additional communication of $|R|$ bits will lead to the recovery of the leftover edges in $R$. Thus, there exists an $\operatorname{LCO}^{(n)}(A)$ of length

$$
\sum_{i=1}^{k}\left|T_{i}\right|-k+|R|=n\left|E^{(1)}\right|-k \text { (bits), }
$$

which establishes the first assertion of (i); also, clearly, $\mathrm{LCO}_{m}^{(n)}(A) \leq n\left|E^{(1)}\right|-k$, thereby proving (ii). To establish the second assertion of (i), it remains to extract a perfect SK from the perfect omniscience obtained using the $\operatorname{LCO}^{(n)}(A)$ above of total length $n\left|E^{(1)}\right|-\mu\left(A, G^{(n)}\right)$ (bits). This is accomplished exactly as in the proof of Theorem 1 , whereby the terminals in $A$ extract a perfect SK $K^{(n)}$ with $\log \left|\mathcal{K}^{(n)}\right|=$ $\mu\left(A, G^{(n)}\right)$.

(iii) Consider an $\operatorname{LCO}^{(n)}(A)=\left(L_{1}, \ldots, L_{m}\right)$ achieving $\operatorname{LCO}_{m}^{(n)}(A)$ with $\left(b_{1}, \ldots, b_{m}\right)$ (bits), respectively. Fix $B \subset$ $\mathcal{M}, B \nsupseteq A$, and consider $\mathcal{S}=\left\{\tilde{x}_{\mathcal{M}}^{n}: \tilde{x}_{j}^{n}=\mathbf{0}\right.$ for every $j \in$ $\left.B^{c}\right\}$ with cardinality $2^{n \sum_{1 \leq i<j \leq m, i \in B}, j \in B} e_{i j}$. For every $k \in$ 
$B^{c} \cap A$ and every $\tilde{x}_{\mathcal{M}}^{n} \in \mathcal{S}$, it holds that $\tilde{x}_{k}^{n}=\mathbf{0}$. Consequently, by the perfect recoverability property of an $\mathrm{LCO}^{(n)}(A)$, such a terminal $k$ must be able to discern all the sequences in $\mathcal{S}$ using only $\left(L_{1}, \ldots, L_{m}\right)$. Note also that for every $\tilde{x}_{\mathcal{M}}^{n} \in \mathcal{S}$ and every $i \in B^{c}$, it follows that $L_{i}\left(\tilde{x}_{i}^{n}\right)=\mathbf{0}$; therefore, the set of all communication messages corresponding to $\mathcal{S}$ has cardinality at most $2^{\sum_{i \in B} b_{i}}$. From the mentioned condition on perfect recoverability at terminal $k \in B^{c} \cap A$ of all sequences in $\mathcal{S}$, it must hold that $2^{\sum_{i \in B} b_{i}} \geq 2^{n \sum_{1 \leq i<j \leq m,(i, j) \in B} e_{i j}}$. Since this argument is valid for every $B \subset \mathcal{M}, B \nsupseteq A$, we have that $\left(b_{1}, \ldots, b_{m}\right) \in \mathcal{I}_{G^{(n)}}(A)$ and, hence, $\operatorname{LCO}_{m}^{(n)}(A)$ is at least

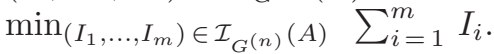

Proof of Corollary 3: The inequality in the Corollary 3 is immediate from (9) and (11). Equality when $A=\mathcal{M}$ relies on Lemma 2 and 3 below; Lemma 2 is a classic result of NashWilliams [11] and Tutte [16] on the maximal size of spanning tree packing of a multigraph, and Lemma 3 [3] provides an upper bound for (standard) SK capacity

Lemma 2: [11], [16] For a multigraph $G=(\mathcal{M}, E)$,

$$
\mu(\mathcal{M}, G)=\left\lfloor\min _{\mathcal{P}} \frac{1}{|\mathcal{P}|-1} \mid\{e \in E: \text { e crosses } \mathcal{P}\} \mid\right\rfloor,
$$

where the minimum is over all partitions $\mathcal{P}$ of $\mathcal{M}$.

Lemma 3: [3] For the multigraph $G=(\mathcal{M}, E)$ associated with the PIN model and for $A \subseteq \mathcal{M}$,

$$
\begin{gathered}
C(A)=|E|-\min _{\left(R_{1}, \ldots, R_{m}\right) \in \mathcal{R}_{G}(A)} \sum_{i=1}^{m} R_{i} \leq \\
\min _{\mathcal{P}} \frac{1}{|\mathcal{P}|-1} \mid\{e \in E: \text { e crosses } \mathcal{P}\} \mid,
\end{gathered}
$$

where the minimum is over all partitions $\mathcal{P}$ of $\mathcal{M}$ such that each atom of $\mathcal{P}$ intersects $A$.

By (6) and 111, $\mathcal{R}_{G^{(n)}}(\mathcal{M}) \supset \mathcal{I}_{G^{(n)}}(\mathcal{M})$ with $G^{(n)}$ and $\mathcal{M}$ in the roles of $G$ and $A$ in (6), it is clear that

$$
\begin{aligned}
& \left\lceil\min _{\left(R_{1}, \ldots, R_{m}\right) \in \mathcal{R}_{G^{(n)}}(\mathcal{M})} \sum_{i=1}^{m} R_{i}\right\rceil \\
& \leq \min _{\left(I_{1}, \ldots, I_{m}\right) \in \mathcal{I}_{G^{(n)}}(\mathcal{M})} \sum_{i=1}^{m} I_{i},
\end{aligned}
$$

noting that the value on the right-side above is an integer.

Then the claimed equality follows since

$$
\begin{aligned}
& \mu\left(\mathcal{M}, G^{(n)}\right) \\
& \leq n\left|E^{(1)}\right|-\min _{\left(I_{1}, \ldots, I_{m}\right) \in \mathcal{I}_{G^{(n)}}(\mathcal{M})} \sum_{i=1}^{m} I_{i} \\
& \leq|n| E^{(1)}\left|-\operatorname{l(R}_{\left(R_{1}, \ldots, R_{m}\right)} \min _{\mathcal{R}_{G^{(n)}}(\mathcal{M})} \sum_{i=1}^{m} R_{i}\right|, \quad \text { by (26) } \\
& \left.\leq\left|\min _{\mathcal{P}} \frac{1}{|\mathcal{P}|-1}\right|\left\{e \in E^{(n)}: e \operatorname{crosses} \mathcal{P}\right\} \mid\right\rfloor \\
& =\mu\left(\mathcal{M}, G^{(n)}\right), \text { by Lemma 2, }
\end{aligned}
$$

Proof of Proposition 4: By remark (ii) after Definition 7 in section III, we have that

$$
\begin{aligned}
\frac{1}{n} \mu\left(A, G^{(n)}\right) & =\frac{1}{n} \max _{\mathcal{T}_{G}^{(n)}(A) \cap \mathbb{Z}^{k}} \sum_{l=1}^{k} T_{l} \\
& =\max _{\mathcal{T}_{G}(A) \cap \frac{1}{n} \mathbb{Z}^{k}} \sum_{l=1}^{k} T_{l} .
\end{aligned}
$$

Since

$$
\lim _{n \rightarrow \infty} \max _{\mathcal{T}_{G}(A) \cap \frac{1}{n} \mathbb{Z}^{k}} \sum_{l=1}^{k} T_{l}=\max _{\mathcal{T}_{G}(A)} \sum_{l=1}^{k} T_{l}=\mu_{f}(A, G),
$$

the assertion follows.

Proof of Theorem 5: The second inequality of the theorem is immediate by Theorem 2 (i) and the definition of $C(A)$.

The proof of the first inequality takes recourse to the following result.

Lemma 4: [8], [6] For a multigraph $G=(\mathcal{M}, E)$ that is Eulerian 6 and $A \subseteq \mathcal{M}$,

$\mu(A, G) \geq\left\lfloor\frac{1}{2} \min _{C \subset \mathcal{M}: C \cap A \neq \emptyset} \mid\left\{e \in E: e\right.\right.$ crosses $\left.\left.C, C^{c}\right\} \mid\right\rfloor$.

Now, for every $n, \mathcal{R}_{G^{(n)}}(A) \supset \mathcal{I}_{G^{(n)}}(A)$, and so

$$
\min _{\mathcal{I}_{G(n)}(A)} \sum_{i=1}^{m} I_{i} \geq \min _{\mathcal{R}_{G}(n)}(A) \sum_{i=1}^{m} R_{i} .
$$

By Lemma 3,

$$
\begin{aligned}
n\left|E^{(1)}\right| & -\min _{\mathcal{R}_{G(n)}(A)} \sum_{1 i=1}^{m} R_{i} \\
& \leq \min _{\mathcal{P}} \frac{1 \mathcal{P} \mid-1}{|\mathcal{P}|} \mid\left\{e \in E^{(n)}: e \text { crosses } \mathcal{P}\right\} \mid \\
& \leq \min _{C \subset \mathcal{M}: C \cap A \neq \emptyset} \mid\left\{e \in E^{(n)}: e \text { crosses } C, C^{c}\right\} \mid .
\end{aligned}
$$

Restricting ourselves to $n$ even, note that $G^{(n)}$ is Eulerian, i.e., each vertex has even degree. Then since the term within \lfloor\rfloor in the right side in Lemma 4 is clearly an integer, we have that

$$
\begin{aligned}
\mu & \left(A, G^{(n)}\right) \\
& \geq \frac{1}{2} \min _{\emptyset \neq C \subset \mathcal{M}: C \cap A \neq \emptyset} \mid\left\{e \in E^{(n)}: e \text { crosses } C, C^{c}\right\} \mid \\
& \geq \frac{1}{2}\left[n\left|E^{(1)}\right|-\min _{\mathcal{R}_{G^{(n)}}(A)} \sum_{i=1}^{m} R_{i}\right], \text { by } \underline{(28)} \\
& =\frac{1}{2}\left[n\left|E^{(1)}\right|-O M N_{G^{(n)}}(A)\right] \\
& =\frac{1}{2} n\left[\left|E^{(1)}\right|-O M N_{G}(A)\right], \text { by (8) } \\
& =\frac{1}{2} n C(A),
\end{aligned}
$$

thereby establishing the left inequality of the theorem.

Proof of Proposition 6: We prove (i) and (ii). The proofs of (iii) and (iv) are similar but simpler, and are omitted.

\footnotetext{
${ }^{6}$ The number of edges incident on each vertex is even.
} 
(i) Similarly as in remark (i) following Definition 7, we note that the right-side of (i) corresponds to a linear program with finite optimum value, and the maximum is attained. Let $\tilde{G}^{*}$, $\left(T_{1}^{*}, \ldots, T_{k_{1}}^{*}\right),\left(T_{1}^{* *}, \ldots, T_{k_{2}}^{* *}\right)$ attain the maximum in the right side of (i), where $\left(T_{1}^{*}, \ldots, T_{k_{1}}^{*}\right)$ and $\left(T_{1}^{* *}, \ldots, T_{k_{2}}^{* *}\right)$ attain the respective maxima in $\mu_{f}\left(A, G^{*}\right)$ and $\mu_{f}\left(\mathcal{M}, G \backslash G^{*}\right)$, with $k_{1}$ (resp. $k_{2}$ ) being the number of all distinct spanning trees in $A$ (resp. $\mathcal{M}$ ) of $G$. Clearly, $\left(T_{1}^{*}, \ldots, T_{k_{1}}^{*}, T_{1}^{* *}, \ldots, T_{k_{2}}^{* *}\right)$ is feasible for $\mu_{f}(A, G)$, noting that a Steiner tree for $A$ of $G$ is either a spanning tree in $A$ or a spanning tree in $\mathcal{M}$.

(ii) Similarly as in the proof of (i), we let $\tilde{G}^{*}$ $\left(R_{1}^{*}, \ldots, R_{m-1}^{*}\right),\left(R_{1}^{* *}, \ldots, R_{m}^{* *}\right)$ attain the minimum in the right side of (ii), where $\left(R_{1}^{*}, \ldots, R_{m-1}^{*}\right)$ and $\left(R_{1}^{* *}, \ldots, R_{m}^{* *}\right)$ attain the respective minima in $O M N_{\tilde{G}^{*}}(A)$ and $O M N_{G \backslash \tilde{G}^{*}}(\mathcal{M})$. Clearly, $\left(R_{1}^{*}+R_{1}^{* *}, \ldots, R_{m-1}^{*}+R_{m-1}^{* *}, R_{m}^{* *}\right)$ is feasible for $O M N_{G}(A)$, thereby proving (ii).

Similar arguments considering the corresponding integer linear programs lead to (iii) and (iv).

Proof of Theorem 7: We shall prove only (i); the proof of (ii) is similar and is omitted.

First, we show that (19) implies (18), i.e.,

$$
\lim _{n \rightarrow \infty} \frac{1}{n} \mu\left(A, G^{(n)}\right) \geq C(A)=|E|-O M N_{G}(A),
$$

(since the reverse inequality always hold by Theorem 5 ). Let a fractional multigraph $\tilde{G}^{*}=\left(A, \tilde{E}^{*}\right)$ achieve the minimum in the right side of (19). Then,

$$
\begin{aligned}
\lim _{n \rightarrow \infty} \frac{1}{n} \mu\left(A, G^{(n)}\right)= & \mu_{f}(A, G), \quad \text { by (15) } \\
\geq & \max _{\tilde{G}} \mu_{f}(A, \tilde{G})+\mu_{f}(\mathcal{M}, G \backslash \tilde{G}), \\
& \quad \text { by Proposition } 6(\mathrm{i}) \\
\geq & \mu_{f}\left(A, \tilde{G}^{*}\right)+\mu_{f}\left(\mathcal{M}, G \backslash \tilde{G}^{*}\right) .
\end{aligned}
$$

Next, because the linear program in the right side of 19 involves a cost and linear constraints with only integer-valued coefficients, $\tilde{G}^{*}=\left(A, \tilde{E}^{*}\right)$ can always be taken to be rational, i.e., all $\tilde{e}_{i j}^{*} \mathrm{~s}$ in $\tilde{E}^{*}$ are rational. Next, let $l$ be the least common multiple of all $\tilde{e}_{i j}^{*}$ s so that $\tilde{G}^{*(l)}=\left(A, \tilde{E}^{*(l)}\right)$ is a multigraph with edge set $\tilde{E}^{*(l)}=\left\{l \tilde{e}_{i j}^{*}, 1 \leq i<j \leq m-1\right\}$. Then,

$$
\begin{aligned}
\mu_{f}\left(A, \tilde{G}^{*}\right) & =\frac{1}{l} \mu_{f}\left(A, \tilde{G}^{*(l)}\right), \text { by 14) } \\
& =\frac{1}{l}\left(\left|\tilde{E}^{*(l)}\right|-O M N_{\tilde{G}^{*(l)}}(A)\right) \\
& =\left|\tilde{E}^{*}\right|-O M N_{\tilde{G}^{*}}(A), \text { by (8); }
\end{aligned}
$$

the second equality is by Proposition 4 and the second assertion of Theorem 5 noting that the vertex set of $\tilde{G}^{*(l)}$ is $A$. By a similar argument, we have that

$$
\mu_{f}\left(\mathcal{M}, G \backslash \tilde{G}^{*}\right)=\left|E \backslash \tilde{E}^{*}\right|-O M N_{G \backslash \tilde{G}^{*}}(\mathcal{M}) .
$$

Substituting (31) and 32) in (30),

$$
\begin{aligned}
\lim _{n \rightarrow \infty} \frac{1}{n} \mu\left(A, G^{(n)}\right) & \\
\geq & \left|\tilde{E}^{*}\right|+\left|E \backslash \tilde{E}^{*}\right| \\
& \quad-\left(O M N_{\tilde{G}^{*}}(A)+O M N_{G \backslash \tilde{G}^{*}}(\mathcal{M})\right) \\
= & |E|-O M N_{G}(A), \text { by 19] }
\end{aligned}
$$

thereby giving 29.

Conversely, to prove that (18) implies (19), i.e.,

$$
O M N_{G}(A) \geq \min _{\tilde{G}} O M N_{\tilde{G}}(A)+O M N_{G \backslash \tilde{G}}(\mathcal{M})
$$

(since the reverse inequality always holds by Proposition 6 (ii)), we can assume similarly as above that $\mu_{f}(A, G)$ is attained by $\left(T_{1}^{*}, \ldots, T_{k}^{*}\right)$ with rational components, where $k=k(G)$ is the number of distinct Steiner trees (for $A$ ) of $G$ (see passage preceding (13)). Next, since $A=\{1, \ldots, m-$ 1) $\subset \mathcal{M}$, the collection of all distinct Steiner trees of (for $A$ ) of $G$, namely $\left\{S_{1}, \ldots, S_{k}\right\}$ can be decomposed as $\mathcal{S}_{1} \sqcup \mathcal{S}_{2}$, where $\mathcal{S}_{1}$ (resp. $\mathcal{S}_{2}$ ) comprises all spanning trees in $A$ (resp. $\mathcal{M})$. Consider the fractional multigraph in $A$ defined by

$$
\left.\tilde{\tilde{G}}^{*}=\left(A, \tilde{\tilde{E}}^{*}\right), \tilde{\tilde{E}}^{*}=\left\{\tilde{\tilde{e}}_{i j}^{*}=\sum_{\substack{l:(i, j) \in S_{l}, S_{l} \in \mathcal{S}_{1}}} T_{l}^{*}, 1 \leq i<j \leq m-1\right\}\right)
$$

Then, it follows that

$$
\mu_{f}(A, G)=\mu_{f}\left(A, \tilde{\tilde{G}}^{*}\right)+\mu_{f}\left(\mathcal{M}, G \backslash \tilde{\tilde{G}}^{*}\right)
$$

since

$$
\begin{aligned}
\mu_{f}(A, G) & =\sum_{l=1}^{k} T_{l}^{*} \\
& =\sum_{l: S_{l} \in \mathcal{S}_{1}} T_{l}^{*}+\sum_{l: S_{l} \in \mathcal{S}_{2}} T_{l}^{*} \\
& \leq \mu_{f}\left(A, \tilde{\tilde{G}}^{*}\right)+\mu_{f}\left(A, G \backslash \tilde{\tilde{G}}^{*}\right),
\end{aligned}
$$

by the definition of $\mu_{f}$; the reverse inequality is always true. Finally, the right side of (18) satisfies

$$
\begin{aligned}
O M N_{\tilde{G}^{*}}(A)+ & O M N_{G \backslash \tilde{G}^{*}}(\mathcal{M}) \\
\leq & O M N_{\tilde{\tilde{G}}^{*}}(A)+O M N_{G \backslash \tilde{\tilde{G}}^{*}}(\mathcal{M}) \\
= & \left(\left|\tilde{\tilde{E}}^{*}\right|-\mu_{f}\left(A, \tilde{\tilde{G}}^{*}\right)\right)+ \\
& \left(\left|E \backslash \tilde{\tilde{E}}^{*}\right|-\mu_{f}\left(\mathcal{M}, G \backslash \tilde{\tilde{G}}^{*}\right)\right), \\
& \quad \text { as in }(\underline{31)}, \quad \text { (32) } \\
= & |E|-\mu_{f}(A, G), \text { by (33) } \\
= & O M N_{G}(A),
\end{aligned}
$$

by (18), (15) and (7).

Proof of Theorem 8: First, we prove (ii), and then (i) by applying (ii) to $G^{(n)}=\left(\mathcal{M}, E^{(n)}\right)$ and taking appropriate limits.

The proof of (ii) entails considering a modification of $G=$ $(\mathcal{M}, E)$ obtained by "edge-splitting" at the helper vertex $m$. Specifically, if $G$ has more than one vertex in $A$ connecting to $m$, then for any two such vertices $u, v \in A$, let $G^{u v}=$ $\left(\mathcal{M}, E^{u v}\right)$ denote the multigraph obtained from $G$ by splitting off the edges $(u, m)$ and $(v, m)$, i.e., by reducing $e_{u m}$ and $e_{v m}$ each by unity and increasing $e_{u v}$ by unity; note that $\left|E^{u v}\right|=$ $|E|-1$.

The following claim, whose proof is relegated to Appendix $\mathrm{B}$, will be used to establish the theorem.

Claim: For a multigraph $G=(\mathcal{M}, E)$,

(a) if $m$ is connected to at most one vertex in $A$ or if there 
exists $\left(I_{1}^{*}, \ldots, I_{m}^{*}\right)$ attaining $I N T_{G}(A)$ with $I_{m}^{*}=0$, then

$$
\mu(A, G)=|E|-I N T_{G}(A) ;
$$

(b) if $m$ is connected to more than one vertex in $A$ and if there exists $\left(I_{1}^{*}, \ldots, I_{m}^{*}\right)$ attaining $I N T_{G}(A)$ with $0<I_{m}^{*} \leq\left\lfloor d_{m} / 2\right\rfloor$, then for $u \in A$ connecting to $m$ there exists $v=v(u) \in A, v \neq u$, also connecting to $m$, such that $\left(I_{1}^{*}, \ldots, I_{m-1}^{*}, I_{m}^{*}-1\right)$ attains $I N T_{G^{u v}}(A)$, and so

$$
|E|-I N T_{G}(A)=\left|E^{u v}\right|-I N T_{G^{u v}}(A) ;
$$

(c) if $m$ is connected to more than one vertex in $A$, then for $u, v \in A$ both connecting to $m$,

$$
\mu(A, G) \geq \mu\left(A, G^{u v}\right) .
$$

In order to prove (ii), we observe first that it holds if the hypothesis of Claim (a) is met. It remains to consider the realm of Claim (b). Let $\left(I_{1}^{*}, \ldots, I_{m}^{*}\right)$ be as in Claim (b). Then we obtain $G_{2}=\left(\mathcal{M}, E_{2}\right)=G^{u v}$ for some $u, v \in A$ connecting to $m$, and with $\left(I_{1}^{*}, \ldots, I_{m}^{*}-1\right)$ attaining $I N T_{G_{2}}(A)$. If $I_{m}^{*}-1=$ 0 or $m$ connects to at most one vertex in $A$ in $G_{2}$, then by (34) (35),

$$
\mu\left(A, G_{2}\right)=\left|E_{2}\right|-I N T_{G_{2}}(A)=|E|-I N T_{G}(A) .
$$

Else, $G_{2}=\left(\mathcal{M}, E_{2}\right)$ is back in the realm of Claim (b), noting that the degree of $m$ in $G_{2}$ is $d_{m}-2$ and $I_{m}^{*}-1 \leq\left\lfloor\left(d_{m}-2\right) / 2\right\rfloor$ as $2 \leq I_{m}^{*} \leq\left\lfloor d_{m} / 2\right\rfloor$.

Thus, we obtain a finite number of multigraphs $G_{1}=$ $G, G_{2}, \ldots, G_{q}$, such that $G_{i}=\left(\mathcal{M}, E_{i}\right)=G_{i-1}^{u v}$ for some $(u, v)=(u, v)(i)$ in $A$, and satisfying

$$
\left|E_{i-1}\right|-I N T_{G_{i-1}}(A)=\left|E_{i}\right|-I N T_{G_{i}}(A), i=2, \ldots, q
$$

and

$$
\mu\left(A, G_{q}\right)=\left|E_{q}\right|-I N T_{G_{q}}(A) .
$$

Using Claim (c) repeatedly,

$$
\begin{aligned}
\mu(A, G) & =\mu\left(A, G_{1}\right) \geq \mu\left(A, G_{q}\right) \\
& =\left|E_{q}\right|-I N T_{G_{q}}(A) . \text { by (37) } \\
& =|E|-I N T_{G}(A)
\end{aligned}
$$

by the repeated use of (36). Then, (ii) is immediate from (38) and Corollary 3.

To establish (i), the hypothesis implies (with a slight abuse of notation) that

$$
\min _{\mathcal{R}_{G}(A) \bigcap\left\{R_{m} \leq d_{m} / 2\right\}} \sum_{i=1}^{m} R_{i}=O M N_{G}(A) .
$$

Pick $\left(R_{1}^{*}, \ldots, R_{m}^{*}\right)$ that attains the left side with all rational components, and let $l$ be the least common multiple of their denominators. Thus, for every integer $n \geq 1,\left(n l R_{1}^{*}, \ldots, n l R_{m}^{*}\right)$ attains $I N T_{G^{(n l)}}(A)$. As $n l R_{m}^{*} \leq n l \frac{\bar{d}_{m}}{2}$, it follows from (ii) that

$$
\begin{aligned}
\mu\left(A, G^{(n l)}\right) & =n l|E|-\operatorname{INT}_{G^{(n l)}}(A) \\
& =n l|E|-n l O M N_{G}(A), \text { by (39). }
\end{aligned}
$$

Upon dividing both sides by $n l$ and taking limits as $n \rightarrow \infty$ (with $l$ fixed), we obtain (i).

\section{Discussion}

We conclude by mentioning several unresolved questions raised by this work.

When all the terminals in $\mathcal{M}$ see to share a perfect SK, i.e., $A=\mathcal{M}$, we see from Theorem 5 that maximal spanning tree packing attains perfect SK capacity; this is no longer true, in general, when $A \subset \mathcal{M}$ (cf. the example in section III.B). However, the single helper model in section III.C possesses the special feature that a Steiner tree for $A$ is a spanning tree for either $A$ or $\mathcal{M}$. In spite of this, it is unresolved whether a maximal Steiner tree packing of $A$ attains perfect SK capacity (i.e., if the second inequality in (16) is tight) or if (12) holds with equality (whereupon the sufficient conditions of Theorem 8 become superfluous). We note that the optimality of maximal spanning tree packing in (12) and (17), constitutes, in effect, a reformulation of the classic graph-theoretic results of NashWilliams [11] and Tutte [16]. A better information theoretic understanding of (12) and (17) is desirable, and might suggest alternative interpretations of related results in combinatorial tree packing.

Perfect SK capacity in Theorem 1 was shown to be achievable by way of the attainment of perfect omniscience at a minimum communication rate $O M N(A)$. However, when $A=\mathcal{M}$, Theorem 5 asserts that maximal spanning tree packing attains capacity; an examination of its proof (cf. Lemma 1) shows the corresponding rate of communication to be $(m-1) C(\mathcal{M})$ which can be less than $O M N(\mathcal{M})$. It remains open to characterize the minimum rate of public communication needed to attain perfect SK capacity.

Maximal Steiner tree packing is guaranteed by Theorem 5 to attain a fraction of at least half of the capacity $C(A)$. What is the best feasible value of this fraction?

Lastly, the design of efficient algorithm for perfect SK generation is largely unexplored.

\section{APPENDIX A: PROOF OF LEMMA 1}

We prove a slightly stronger result that there exists an LC whose null space comprises only the all-zero and the all-one strings (corresponding to the edges in $T$ being labelled all zero or all one) which clearly enables every terminal in $V$ to recover all the edges of $T$. We prove the claim by induction. When $|T|=2$, say, with $T=\left\{e_{1}=\left(v_{1}, v_{2}\right), e_{2}=\left(v_{2}, v_{3}\right)\right\}$, then $e_{1}+e_{2} \bmod 2$ constitutes an LC whose null space is $\{(00),(11)\}$. Next, suppose the claim is true for all trees with $k-1$ edges, $k \geq 3$. Given a tree with $k$ edges, pick an end vertex $v_{k+1}$ of the tree (a vertex with degree one), and let $v_{k}$ be the sole vertex connecting to $v_{k+1}$. Then $G=\left(V, T^{\prime} \bigcup\left\{\left(v_{k}, v_{k+1}\right)\right\}\right)$, and $G^{\prime}=\left(V \backslash\left\{v_{k+1}\right\}, T^{\prime}\right)$ is a subtree of $G$. By the induction hypothesis, there exists an LC for $G^{\prime}$, say, $F\left(T^{\prime}\right)$ of length $k-2$ (bits) and whose null space is $\left\{\mathbf{0}^{k-1}, \mathbf{1}^{k-1}\right\}$. Let $v_{k-1}$ be another vertex connecting to $v_{k}$ and let $e_{k-1}=\left(v_{k-1}, v_{k}\right)$ and $e_{k}=\left(v_{k}, v_{k+1}\right)$. Then, consider $\left\{F\left(T^{\prime}\right), e_{k-1}+e_{k}\right\}$ as an LC of $G$ of length $k-1$. It is now clear that the null space of this LC is $\left\{\mathbf{0}^{k}, \mathbf{1}^{k}\right\}$. 


\section{APPENDIX B: Proof of Claim In (THE PROOF OF) THEOREM 8}

(a) Let $G_{A}=\left(A, E_{A}\right)$ denote a subgraph of $G$ in $A$, where $E_{A} \subset E$ consists only of those edges in $E$ whose both end vertices lie in $A$. Clearly,

$$
\begin{aligned}
|E|-I N T_{G}(A) \geq & \mu(A, G) \geq \mu\left(A, G_{A}\right) \\
= & \left|E_{A}\right|-I N T_{G_{A}}(A), \\
& \quad \text { by Corollary } 3 \text { with } \mathcal{M}=A \\
= & |E|-\left(d_{m}+I N T_{G_{A}}(A)\right) .
\end{aligned}
$$

Thus, it suffices to show that

$$
d_{m}+I N T_{G_{A}}(A) \leq I N T_{G}(A) .
$$

Consider first the case where $\left(I_{1}^{*}, \ldots, I_{m-1}^{*}, 0\right)$ attains $I N T_{G}(A)$. Without loss of generality, let $\{1, \ldots, a\}, a \leq$ $m-1$, be the set of vertices in $A$ connecting to $m$. For any $v \in\{1, \ldots, a\}$, since $\{v, m\} \nsupseteq A$, we have that $I_{v}^{*}+I_{m}^{*}=$ $I_{v}^{*} \geq e_{v m}$ (see (11)). Consequently, since $d_{m}=\sum_{u=1}^{a} e_{u m}$, we see that $\left(I_{1}^{*}-e_{1 m}, \ldots, I_{a}^{*}-e_{a m}, I_{a+1}^{*}, \ldots, I_{m-1}^{*}\right)$, with components summing to $\operatorname{INT}_{G}(A)-d_{m}$ is feasible for $I N T_{G_{A}}(A)$. Thus, $I N T_{G}(A)-d_{m} \geq I N T_{G_{A}}(A)$, establishing (B-1). A nearly identical argument would show that B-1 holds too for the case when at most vertex 1 is connected to $m$, and is omitted.

(b) Consider any $G^{u v}=\left(\mathcal{M}, E^{u v}\right)$ as in the second paragraph of the proof of Theorem 8 , and let $\left(I_{1}^{* *}, \ldots, I_{m}^{* *}\right)$ attain $I N T_{G^{u v}}(A)$. Then, $\left(I_{1}^{* *}, \ldots, I_{m-1}^{* *}, I_{m}^{* *}+1\right)$ is feasible for $\operatorname{INT}_{G}(A)$, so that

$$
\operatorname{INT}_{G}(A) \leq I N T_{G^{u v}}(A)+1 .
$$

Without loss of generality, let $\{1, \ldots, a\}$ be as in the proof of Claim (a). To prove Claim (b), it suffices to show for $u=1$ that there exists $v \in\{2, \ldots, a\}$ such that $\left(I_{1}^{*}, \ldots, I_{m-1}^{*}, I_{m}^{*}-\right.$ 1 ) is feasible for $I N T_{G^{1 v}}(A)$ if $0<I_{m}^{*} \leq\left\lfloor\frac{d_{m}}{2}\right\rfloor$. This would mean that

$$
I N T_{G}(A)-1 \geq I N T_{G^{1 v}}(A) .
$$

which, together with the observation that $|E|-1=\left|E^{1 v}\right|$, establishes Claim (b). To this end, referring to (11), for $B \subseteq$ $\mathcal{M}$, set

$$
e_{G}(B) \triangleq \sum_{1 \leq i<j \leq m, i \in B, j \in B} e_{i j}, \quad e_{G}(\emptyset) \triangleq 0,
$$

and let

$$
\mathcal{B}=\left\{\begin{array}{l}
B, \emptyset \neq B \subset \mathcal{M}, B \nsupseteq A, \\
\sum_{i \in B} I_{i}^{*}=e_{G}(B)
\end{array}\right\} .
$$

We make the following

Claim (d): For $u=1$, there exists $v \in\{2, \ldots, a\}$ connecting to $m$ with the properties that

a) for $B \in \mathcal{B}$ such that $1 \notin B, m \in B$, it holds that $v \in B$;

b) for $B \in \mathcal{B}$ such that $1 \in B, m \notin B$, it holds that $v \notin B$.

Then, with the choice of $v$ as in the Claim (d), a simple check of all the possibilities for $B$ (in $\mathcal{B}$ or in $\mathcal{B}^{c}$ ) that are feasible in 11), shows that $\left(I_{1}^{*}, \ldots, I_{m-1}^{*}, I_{m}^{*}-1\right)$ is feasible for $\operatorname{INT}_{G^{1 v}}(A)$, thereby establishing (B-3) (and hence Claim (b)).
It only remains to establish Claim (d). We first state the following facts with accompanying proofs.

Fact 1: For $B_{1}, B_{2} \subset \mathcal{M}, e_{G}\left(B_{1}\right)+e_{G}\left(B_{2}\right) \leq$ $e_{G}\left(B_{1} \cup B_{2}\right)+e_{G}\left(B_{1} \cap B_{2}\right)$. This holds by observing that $e_{G}\left(B_{1} \cup B_{2}\right)+e_{G}\left(B_{1} \cap B_{2}\right)-e_{G}\left(B_{1}\right)-e_{G}\left(B_{2}\right)=$ $\sum_{1 \leq i<j \leq m, i \in B_{1} \backslash B_{2}, j \in B_{2} \backslash B_{1} \text { or } i \in B_{2} \backslash B_{1}, j \in B_{1} \backslash B_{2}} e_{i j} \geq 0$.

Fact 2: For $B_{1}, B_{2} \in \mathcal{B}$ with $B_{1} \cup B_{2} \nsupseteq A$, it holds that $B_{1} \cup B_{2}$ and $B_{1} \cap B_{2}$ are both in $\mathcal{B}$. To see this, note first that

$$
\begin{aligned}
\sum_{i \in B_{1} \cup B_{2}} I_{i}^{*} & =\sum_{i \in B_{1}} I_{i}^{*}+\sum_{i \in B_{2}} I_{i}^{*}-\sum_{i \in B_{1} \cap B_{2}} I_{i}^{*} \\
& =e_{G}\left(B_{1}\right)+e_{G}\left(B_{2}\right)-\sum_{i \in B_{1} \cap B_{2}} I_{i}^{*} \\
& \leq e_{G}\left(B_{1}\right)+e_{G}\left(B_{2}\right)-e_{G}\left(B_{1} \cap B_{2}\right) \\
& \leq e_{G}\left(B_{1} \cup B_{2}\right), \text { by Fact } 1 .
\end{aligned}
$$

Also, $\sum_{B_{1} \cup B_{2}} I_{i}^{*} \geq e_{G}\left(B_{1} \cup B_{2}\right)$, since $B_{1} \cup B_{2} \nsupseteq A$ is feasible in 111). The fact follows.

Fact 3: For $B \subseteq \mathcal{M}$, let $D_{m}(B)$ denote the total number of edges connecting $m$ to all the vertices in $B \cap A$. Then, for $B \in \mathcal{B}$, if $m \in B$ then $D_{m}(B) \geq I_{m}^{*}$, and if $m \notin B$ then $D_{m}(B) \leq I_{m}^{*}$. To see this, consider first the case $m \in$ $B \in \mathcal{B}$. As $\{m\} \notin \mathcal{B}$ (since $I_{m}^{*}>0$ ), we have $B \cap A \neq \emptyset$. Since $B \in \mathcal{B}, \sum_{i \in B} I_{i}^{*}=e_{G}(B \cap A)+D_{m}(B)$. Also, since $B \cap A \neq \emptyset$ is feasible in (11), $\sum_{i \in B \cap A} I_{i}^{*} \geq e_{G}(B \cap A)$. Subtracting the latter from the former gives $I_{m}^{*} \leq D_{m}(B)$. The second assertion of the fact is proved similarly.

Fact 4: The intersection of all $B s$ in $\mathcal{B}$ satisfying $1 \notin$ $B, m \in B$, when nonempty, is also in $\mathcal{B}$. The union of all $B s$ in $\mathcal{B}$ satisfying $1 \in B, m \notin B$, when nonempty, is also in $\mathcal{B}$.

The first assertion in Fact 4 is obtained by observing that the union of all $B s$ in $\mathcal{B}$ with $1 \notin B, m \in B$, does not contain $A$, and by a repeated use of Fact 2 . The second assertion would follow similarly by Fact 2 if the union of all $B s$ in $\mathcal{B}$ with $1 \in B, m \notin B$, is strictly contained in $A$. Suppose not; then this union is exactly $A$. The ensuing contradiction can be seen, for instance, with $B_{1}, B_{2}$ as above with $B_{1} \cup B_{2}=A$. Then

$$
\begin{aligned}
d_{m} & =D_{m}(A)=D_{m}\left(B_{1} \cup B_{2}\right) \\
& =D_{m}\left(\left(B_{1} \backslash B_{2}\right) \cup\left(B_{1} \cap B_{2}\right) \cup\left(B_{2} \backslash B_{1}\right)\right) \\
& =D_{m}\left(B_{1} \backslash B_{2}\right)+D_{m}\left(B_{1} \cap B_{2}\right)+D_{m}\left(B_{2} \backslash B_{1}\right) \\
& =D_{m}\left(B_{1}\right)+D_{m}\left(B_{2}\right)-D_{m}\left(B_{1} \cap B_{2}\right) \\
& \leq I_{m}^{*}+I_{m}^{*}-1, \text { by Fact } 3 \text { and } 1 \in B_{1} \cap B_{2} \\
& \leq 2\left\lfloor\frac{d_{m}}{2}\right\rfloor-1, \\
& \quad \text { by the assumption } I_{m}^{*} \leq\left\lfloor\frac{d_{m}}{2}\right\rfloor \\
< & d_{m},
\end{aligned}
$$

a contradiction.

Finally, to prove Claim (d), let $B^{\prime}$ (resp. $B^{\prime \prime}$ ) represent the intersection (resp. union), when nonempty, in Fact 4. It suffices now to show that there exists $v \in B^{\prime} \cap A$ (when $B^{\prime} \neq \emptyset$ ) such 
that $v \notin B^{\prime \prime}$ and $v$ connects to $m$; this follows from

$$
\begin{aligned}
D_{m}\left(B^{\prime} \backslash B^{\prime \prime}\right) & =D_{m}\left(B^{\prime}\right)-D_{m}\left(B^{\prime} \cap B^{\prime \prime}\right) \\
& =D_{m}\left(B^{\prime}\right)-\left(D_{m}\left(B^{\prime \prime}\right)-D_{m}\left(B^{\prime \prime} \backslash B^{\prime}\right)\right) \\
\geq & I_{m}^{*}-\left(I_{m}^{*}-1\right), \\
& \quad \text { by Fact } 3 \text { and } 1 \in B^{\prime \prime} \backslash B^{\prime} \\
= & 1 .
\end{aligned}
$$

Then, any $B$ as in Claim (d)(a) must contain $B^{\prime}$ and hence the $v$ above. On the other hand, any $B$ as in Claim (d)(b) must be contained in $B^{\prime \prime}$ and so cannot contain the $v$ above. The cases $B^{\prime}=\emptyset$ or $B^{\prime \prime}=\emptyset$ are handled trivially.

(c) Let $G^{u v}=\left(\mathcal{M}, E^{u v}\right)$ and suppose that $T_{1} \sqcup \ldots \sqcup T_{k} \subseteq$ $E^{u v}$ attain $\mu\left(A, G^{u v}\right)$. If $E^{u v} \backslash\left\{\sqcup_{i=1}^{k} T_{i}\right\}$ contains at least one edge connecting $(u, v)$, then $\left\{T_{1}, \ldots, T_{k}\right\}$ is also a Steiner tree packing of $G=(\mathcal{M}, E)$, so that $\mu(A, G) \geq \mu\left(A, G^{u v}\right)$. Else, let $T_{1}$, say, be the Steiner tree that contains an edge connecting $u, v$ that emerged by splitting off $(u, m)$ and $(v, m)$ of $G=(\mathcal{M}, E)$. Then, $\left\{T_{1} \backslash\{(u, v)\}\right\} \cup\{(u, m),(v, m)\}$ is A-connected and hence contains a Steiner tree $T_{1}^{\prime}$ for $A$ in $G=(\mathcal{M}, E)$ that corresponds to $T_{1}$; clearly, again $\mu(A, G) \geq$ $\mu\left(A, G^{u v}\right)$.

\section{ACKNOWLEDGEMENT}

The authors thank Chunxuan Ye, Alexander Barg and Alex Reznik for very helpful discussions.

\section{REFERENCES}

[1] R. Ahlswede and I. Csiszár, "Common randomness in information theory and cryptography, Part I: Secret sharing," IEEE Trans. Inf. Theory, vol. 39, pp. 1121-1132, July 1993.

[2] I. Csiszár and P. Narayan, "Common randomness and secret key generation with a helper," IEEE Trans. Inf. Theory, vol. 46, pp. 344366, Mar. 2000.

[3] I. Csiszár and P. Narayan, "Secrecy capacities for multiple terminals," IEEE Trans. Inf. Theory, vol. 50, pp. 3047-3061, Dec. 2004.
[4] I. Csiszár and P. Narayan, "Secrecy capacities for multiterminal channel models," Special Issue of the IEEE Trans. Inf. Theory on Information Theoretic Security, vol. 54, pp. 2437-2452, June 2008.

[5] M. Grötschel, A. Martin and R. Weismantel, "Packing Steiner trees: A cutting plane algorithm and computational results," Math. Programming, vol. 72, pp. 125-145, Feb. 1996.

[6] M. Kriesell, "Edge-disjoint trees containing some given vertices in a graph," J. of Combinatorial Theory, Series B, vol. 88, pp. 5365, 2003.

[7] Z. Li, B. Li and L. C. Lau, "On achieving maximum multicast throughput in undirected networks," IEEE Trans. Inf. Theory, vol. 52, pp. 24672485, June 2006.

[8] L. Lovász, "On some connectivity properties of Eulerian graphs," Acta Math. Akad. Sci. Hung., vol. 28, pp. 129-139, 1976.

[9] U. M. Maurer, "Provably secure key distribution based on independent channels," presented at the IEEE Workshop Inf. Theory, Eindhoven, The Netherlands, 1990.

[10] U. M. Maurer, "Secret key agreement by public discussion from common information," IEEE Trans. Inf. Theory, vol. 39, pp. 733-742, May 1993

[11] C. St. J. A. Nash-Williams, "Edge disjoint spanning trees of finite graphs," J. London Math. Soc., 36, pp. 445-450, 1961.

[12] S. Nitinawarat, C. Ye, A. Barg, P. Narayan and A. Reznik, "Secret key generation for a pairwise independent network model," in Proc. 2008 IEEE Int. Symp. on Inf. Theory, Toronto, Ontario, Canada, pp. 10151019 .

[13] S. Nitinawarat, C. Ye, A. Barg, P. Narayan and A. Reznik, "Common randomness, multiuser secrecy and tree packing," in Proc. 46th Annu. Allerton Conf. on Commun., Control, and Computing, Monticello, IL, 2008, pp. 217-220.

[14] S. Nitinawarat, C. Ye, A. Barg, P. Narayan and A. Reznik, "Secret key generation for a pairwise independent network model," IEEE Trans. Inf. Theory, in review.

[15] A. Schrijver, Theory of Linear and Integer Programming, New York: Wiley, 1986.

[16] W. T. Tutte, "On the problem of decomposing a graph into $n$ connected factors," J. London Math. Soc., 36, pp. 221-230, 1961.

[17] Y. Wu, K. Jain and S.-Y. Kung, "A unification of network coding and tree-packing (routing) theorems," IEEE Trans. Inf. Theory, vol. 52, pp. 2398-2409, June 2006.

[18] C. Ye and P. Narayan, "Secret key and private key constructions for simple multiterminal source models," in Proc. 2005 IEEE Int. Symp. on Inf. Theory, Adelaide, Australia, pp. 2133-2137.

[19] C. Ye and A. Reznik, "Group secret key generation algorithms," in Proc. 2007 IEEE Int. Symp. on Inf. Theory, Nice, France, pp. 2596-2600.

[20] C. Ye, A. Reznik and Y. Shah, "Extracting secrecy from jointly Gaussian random variables," in Proc. 2006 IEEE Int. Symp. on Inf. Theory, Seattle, pp. 2593-2597. 